\title{
ON THE CONVERGENCE OF AN APPROXIMATION SCHEME FOR THE VISCOSITY SOLUTIONS OF THE BELLMAN EQUATION ARISING IN A STOCHASTIC OPTIMAL CONTROL PROBLEM*
}

BY

\author{
TEODOR HAVÂRNEANU
}

\begin{abstract}
The paper presents an Euler step approximation scheme for the viscosity solution of the dynamic programming equation for a stochastic control problem with infinite horizon. We established the rate of convergence of this scheme.

Mathematics Subject Classification 2000: 93E20, 49L20, 49L25, 60H10.

Key words: stochastic optimal control, Bellman equations, viscosity solution.
\end{abstract}

\section{Introduction and preliminary results}

At the beginning of ' 80 , using the viscosity solutions theory, several approximation results for the Bellman equation in the deterministic case were obtained (see [1],[2],[4],[5]). In [6] we proposed an Euler step approximation scheme (similar to those from [1],[3],[5]) for the viscosity solution of the Bellman equation of a stochastic optimal control problem and we proved its convergence. In the present paper we give a result concerning the rate of convergence for the approximation scheme presented in [6] and also we improve the convergence result there.

Let us consider the following stationary second order Bellman partial differential equation

$$
\lambda u(x)+\sup _{a \in A}[-\nabla u(x) \cdot b(x, a)-f(x, a)]-\frac{\varepsilon}{2} \Delta u(x)=0, x \in \mathbb{R}^{n},
$$

* Research supported by Grant CNCSIS $1373 / 2007$ and by Grant FP7-ITN, G.A.2/3841/2008. 
where $u(\cdot)$ is the unknown function, $A \subset \mathbb{R}^{m}$ is a compact set, $b: \mathbb{R}^{n} \times A \rightarrow$ $\mathbb{R}^{n}$ and $f: \mathbb{R}^{n} \times A \rightarrow \mathbb{R}^{n}$ are two given functions, $\lambda>0$ and $\varepsilon>0$ are two given constants, and "." stands for the inner product in $\left(\mathbb{R}^{n},\|\cdot\|\right)$.

We suppose that the functions $b$ and $f$ satisfy the following hypotheses: There exist the positive constants $L_{1} \in(0, \lambda), L_{2}$, and $M$ such that

(i) $\quad\|b(x, a)-b(y, a)\| \leq L_{1}\|x-y\|, x, y \in \mathbb{R}^{n}, a \in A$

(ii) $\quad|f(x, a)-f(y, a)| \leq L_{2}\|x-y\|, x, y \in \mathbb{R}^{n}, a \in A$

(iii) $\quad\|b(x, a)\| \leq M,|f(x, a)| \leq M, x \in \mathbb{R}^{n}, a \in A$.

In [6] we have proved that equation (1.1) has a unique viscosity solution which is the value functions of the following stochastic control problem with infinite horizon.

Let $\left(\Omega, \mathcal{F}, P,\left(\mathcal{F}_{t}\right)_{t \geq 0}\right)$ be a fixed complete stochastic basis which means that $(\Omega, \mathcal{F}, P)$ is a complete probability space and $\left(\mathcal{F}_{t}\right)_{t \geq 0}$ is an increasing family of sub- $\sigma$-algebras of $\mathcal{F}$ such that $\mathcal{F}_{0}$ contains every $P$-negligible subset of $\Omega$. Also we consider a standard $n$-dimensional $\mathcal{F}_{t}$-adapted Wiener process $\{w(t), t \geq 0\}$ and

$$
\begin{aligned}
\mathcal{A}=\{a:[0,+\infty) \times \Omega \rightarrow A ; a(\cdot, \cdot) \text { is measurable } \\
\\
\text { and } \left.a(t, \cdot) \text { is } \mathcal{F}_{t}-\text { measurable, } t \geq 0\right\} .
\end{aligned}
$$

Remark 1.1. In the following we shall consider that the $\sigma$-algebra $\mathcal{F}_{t}$ is generated by $\{w(s), 0 \leq s \leq t\}$ for $t \geq 0$.

Now we present the optimal control problem:

Minimize

$$
J(x, a)=E\left[\int_{0}^{\infty} f(x(t), a(t)) e^{-\lambda t} d t\right],
$$

where $E$ stands for the average, $a \in \mathcal{A}$ and $(x(t), a(t))$ satisfies the state equation

$$
\left\{\begin{array}{l}
d x(t)=b(x(t), a(t)) d t+\sqrt{\varepsilon} d w(t), \varepsilon>0 \\
x(0)=x \in \mathbb{R}^{n}
\end{array}\right.
$$

We define

$$
J(x)=\inf _{a \in \mathcal{A}} J(x, a), x \in \mathbb{R}^{n} .
$$


As we have mentioned before, the function given by (1.5) is the unique viscosity solution of (1.1). For the reader's convenience we shall give the definition of the viscosity solution for (1.1).

Definition $1.1([7])$. The function $u \in C\left(\mathbb{R}^{n}\right)$ is called viscosity subsolution (supersolution) of (1.1) iff for every $\varphi \in C^{2}\left(\mathbb{R}^{n}\right)$ for which $(u-\varphi)$ has a (strict) local maximum (minimum) in $x_{0}$ we have

$$
\lambda u\left(x_{0}\right)+\sup _{a \in A}\left[-\nabla \varphi\left(x_{0}\right) \cdot b\left(x_{0}, a\right)-f\left(x_{0}, a\right)\right]-\frac{\varepsilon}{2} \Delta \varphi\left(x_{0}\right) \leq 0 \quad(\geq 0) .
$$

A function $u(\cdot)$ which is at the same time a viscosity subsolution and a viscosity supersolution is called viscosity solution.

In the next we present the approximation scheme ([6]).

Let $h>0$ and $t_{j}=j h, j=0,1,2, \ldots$. Corresponding to each $h>0$ we consider the set of discrete controls:

$$
\begin{aligned}
\mathcal{A}_{h}= & \left\{a \in \mathcal{A} ; a \text { is constant on }\left[t_{j}, t_{j+1}\right), j=0,1,2, \ldots,\right. \text { i.e. } \\
& \left.a(s)=a_{j}(\cdot) \in L^{2}(\Omega, A), \text { for } s \in\left[t_{j}, t_{j+1}\right), j=0,1,2, \ldots\right\} .
\end{aligned}
$$

Now we define recursively the sequence $\left\{x_{j}\right\}_{j \geq 0}$ (the approximation of $(1.4))$

$$
\left\{\begin{array}{l}
x_{0}=x \\
x_{j+1}=x_{j}+h b\left(x_{j}, a_{j}\right)+\sqrt{\varepsilon}\left(w\left(t_{j+1}\right)-w\left(t_{j}\right)\right), j \in \mathbb{N}
\end{array}\right.
$$

where $a \in \mathcal{A}_{h}\left(a_{j}=a(s), s \in\left[t_{j}, t_{j+1}\right)\right)$. Also we consider the following discretization of the functional (1.3)

$$
J_{h}(x, a)=h E \sum_{j=0}^{\infty}(1-\lambda h)^{j} f\left(x_{j}, a_{j}\right),
$$

were $a \in \mathcal{A}_{h}$ and $\left\{x_{j}\right\}_{j \geq 0}$ verifies (1.7).

Now we define the approximate solution of (1.1) as

$$
v_{h}(x)=\inf _{a \in \mathcal{A}_{h}} J_{h}(x, a)=\inf _{a \in \mathcal{A}_{h}} E \sum_{j=0}^{\infty}(1-\lambda h)^{j} f\left(x_{j}, a_{j}\right) .
$$


For $h \in\left(0 ; \frac{1}{\lambda}\right]$ we consider the following approximate equation of (1.1)

$$
\begin{aligned}
& u_{h}(x)+\sup _{a \in A} E\left[-(1-\lambda h) u_{h}(x+h b(x, a)+\sqrt{\varepsilon} w(h))+f(x, a)\right]=0, \\
& x \in \mathbb{R}^{n}
\end{aligned}
$$

In the next we shall prove a dynamic programming principle for the discrete optimal control problem (1.9), (1.7).

Theorem 1.1. For each $x \in \mathbb{R}^{n}$ we have

$$
v_{h}(x)=\inf _{a_{0}} E\left[h f\left(x, a_{0}\right)+(1-\lambda h) v_{h}\left(x_{1}\right)\right],
$$

where $a_{0} \in L^{2}(\Omega, A)$ is $\mathcal{F}_{0}$ - measurable and $x_{1}$ is given by $(1.7)$ with $x_{0}=x$.

Proof. Using (1.9) and (1.8) we obtain that for each $\nu>0$ there exists $a^{\nu} \in \mathcal{A}_{h}$ such that

$$
\begin{aligned}
& v_{h}(x)+\nu \geq J_{h}\left(x, a^{\nu}\right)=h E f\left(x, a_{0}^{\nu}\right)+(1-\lambda h) h E \\
& \sum_{j=0}^{\infty}(1-\lambda h)^{j} f\left(x_{j+1}^{\nu}, a_{j+1}^{\nu}\right) \geq h E f\left(x, a_{0}^{\nu}\right)+(1-\lambda h) E v_{h}\left(x_{1}^{\nu}\right),
\end{aligned}
$$

where $\left\{x_{j}^{\nu}\right\}_{j \in \mathbb{N}}$ and $\left\{a_{j}^{\nu}\right\}_{j \in \mathbb{N}}$ verify (1.7). Taking the infimum in (1.12) we obtain

$$
v_{h}(x)+\nu \geq \inf _{a \in \mathcal{A}_{h}} E\left[h E f\left(x, a_{0}\right)+(1-\lambda h) v_{h}\left(x_{1}\right)\right],
$$

for every $\nu>0$. For every $\nu>0$ there exist $\widetilde{a}^{\nu}, \widehat{a}^{\nu} \in \mathcal{A}_{h}$ such that

$$
\begin{aligned}
& \inf _{a \in \mathcal{A}_{h}} E\left[h f(x, a)+(1-\lambda h) v_{h}\left(x_{1}\right)\right]+\nu \\
& \geq E\left[h f\left(x, \widetilde{a}_{0}^{\nu}\right)+(1-\lambda h) v_{h}\left(\widetilde{x}_{1}^{\nu}\right)\right] \\
& \geq E\left[h f\left(x, \widetilde{a}_{0}^{\nu}\right)+(1-\lambda h) h \sum_{j=0}^{\infty}(1-\lambda h)^{j} f\left(\widehat{x}_{j}^{\nu}, \widehat{a}_{j}^{\nu}\right)\right]-\nu,
\end{aligned}
$$

where $\left\{\widetilde{x}_{j}^{\nu}\right\}_{j \in \mathbb{N}},\left\{\widetilde{a}_{j}^{\nu}\right\}_{j \in \mathbb{N}}$ satisfy (1.7) with $\widetilde{x}_{0}^{\nu}=x$, and $\left\{\widehat{x}_{j}^{\nu}\right\}_{j \in \mathbb{N}},\left\{\widehat{a}_{j}^{\nu}\right\}_{j \in \mathbb{N}}$ satisfy (1.7) with $\widehat{x}_{0}^{\nu}=\widetilde{x}_{1}^{\nu}$.

Let $\bar{a}^{\nu}=\left\{\bar{a}_{j}^{\nu}\right\}_{j \in \mathbb{N}}$ and $\bar{x}^{\nu}=\left\{\bar{x}_{j}^{\nu}\right\}_{j \in \mathbb{N}}$ defined by

$$
\left\{\begin{array}{l}
\bar{a}_{0}^{\nu}=\widetilde{a}_{0}^{\nu}, \\
\bar{a}_{j}^{\nu}=\widehat{a}_{j-1}^{\nu}, \quad j \geq 1
\end{array}, \quad\left\{\begin{array}{l}
\bar{x}_{0}^{\nu}=\widetilde{x}_{0}^{\nu}=x, \\
\bar{x}_{j}^{\nu}=\widehat{x}_{j-1}^{\nu}, \quad j \geq 1
\end{array}\right.\right.
$$


Taking into account (1.13), (1.14), (1.15) and (1.8) it results

$$
\inf _{a \in \mathcal{A}_{h}} E\left[h f\left(x, a_{0}\right)+(1-\lambda h) v_{h}\left(x_{1}\right)\right]+2 \nu \geq J_{h}\left(\bar{a}^{\nu}, x\right) \geq v_{h}(x) .
$$

Because $\nu>0$ is arbitrarily chosen, the relations (1.14) and (1.16) imply (1.11).

In the next, for the reader's convenience, we shall present some results (see also [6]) which we need in evaluation of the rate of convergence of our scheme.

Theorem $1.2([6])$. In the hypotheses $(\mathrm{H})$ the $v_{h}$ given by $(1.9)$ is the unique bounded solution of (1.10).

Sketch of the proof. Using Theorem 1.1 we get that $v_{h}$ verifies

$$
\begin{aligned}
v_{h}(x) & +\sup _{a_{0}} E\left[-(1-\lambda h) v_{h}\left(x+h b\left(x, a_{0}\right)\right)\right. \\
& \left.+\sqrt{\varepsilon} w(h)-h f\left(x, a_{0}\right)\right]=0, \quad x \in \mathbb{R}^{n},
\end{aligned}
$$

where $a_{0} \in L^{2}(\Omega, A)$ and $a_{0}$ is $\mathcal{F}_{0}$-measurable (see the definitions (1.2) and $(1.6))$.

Taking into account the fact that $\mathcal{F}_{0}$ and $\mathcal{F}_{h}$ are independent (see Remark 1.1) we may write the average in (1.14) as a double integral and so we obtain

$$
\begin{aligned}
& v_{h}(x)+\sup _{a_{0} \in L^{2}\left(\Omega_{1}, A\right)} \int_{\Omega_{1}}\left\{\int _ { \Omega } \left[-(1-\lambda h) v_{h}\left(x+b\left(x, a_{0}\left(\omega_{1}\right)\right)\right.\right.\right. \\
& \left.\left.+\sqrt{\varepsilon} \omega(h)(\omega))-h f\left(x, a_{0}\left(\omega_{1}\right)\right)\right] d P(\omega)\right\} d P\left(\omega_{1}\right)=0,
\end{aligned}
$$

where $\Omega_{1}$ is $\Omega$ but we put subscript 1 to be more clear. Using boundness of $v_{h}$ (which is evident from (1.8)), from the relation (1.18) we obtain that $v_{h}$ is a solution of (1.10).

To prove the uniqueness, we suppose that there exist two solutions $u_{1}$ and $u_{2}$ of (1.10) and subtracting the two relations (1.10) which they verify, using their boundness and some calculus, it results

$$
\left|u_{1}(x)-u_{2}(x)\right| \leq(1-\lambda h) \sup _{y \in \mathbb{R}^{n}}\left|u_{1}(y)-u_{2}(y)\right|, x \in \mathbb{R}^{n},
$$

relation which proves the uniqueness because $0<h \leq \frac{1}{\lambda}$. 
Theorem $1.3([6])$. In the hypotheses $(\mathrm{H})$ the function $v_{h}(\cdot)$ converges to $J(\cdot)$ locally uniformly on $\mathbb{R}^{n}$ as $h$ tends to $O$.

Sketch of the proof. Using (1.9) and the hypotheses on $b, f$, and $\lambda$, it results

$$
\begin{aligned}
& \sup _{x \in \mathbb{R}^{n}}\left|v_{h}(x)\right| \leq \frac{M}{\lambda} \\
& \left|v_{h}(x)-v_{h}\left(x^{\prime}\right)\right| \leq \frac{L_{2}}{\lambda-L_{1}}\left\|x-x^{\prime}\right\|, \text { for } x, x^{\prime} \in \mathbb{R}^{n} \text { and } h \in\left(0, \frac{1}{\lambda}\right] .
\end{aligned}
$$

From the Ascoli-Arzela theorem we obtain that there exists a bounded Lipschitz continuous function $u(\cdot)$ such that $v_{h}(\cdot)$ converges to $u(\cdot)$ locally uniformly on $\mathbb{R}^{n}$ as $h$ tends to 0 .

In the following we shall prove that $u(\cdot)$ is a viscosity subsolution of (1.1) (in the same manner one can prove that it is also a viscosity supersolution). We shall present this proof in more details because we consider that it is interesting and useful for the reader.

Let $\varphi \in C_{b}^{2}\left(\mathbb{R}^{n}\right)$ (a bounded function of class $C^{2}$ ), $x_{0}$ a strict local maximum point of $(u-\varphi), \bar{B}\left(x_{0}, r\right)=\left\{x \in \mathbb{R}^{n} \mid\left\|x-x_{0}\right\| \leq r\right\}$ and $x_{0}^{h}$ a global maximum point of $\left(v_{h}-\varphi\right)$ on $\bar{B}\left(x_{0}, r\right)$. The sequence $\left\{x_{0}^{h}\right\}_{h>0}$ converges to $x_{0}$ as $h$ tends to 0 (see [3]) and so we can choose $h_{1}>0$ such that for every $h \in\left(0, h_{1}\right)$ we have

$$
\left\|x_{0}^{h}-x_{0}\right\| \leq \frac{r}{3}, h M \leq \frac{r}{3} .
$$

Let

$$
\Omega_{r}=\left\{\omega \in \Omega ;\|w(h)(\omega)\| \leq \frac{r}{3}\right\}
$$

It is well-known (see [2]) that

$$
P\left(\Omega \backslash \Omega_{r}\right) \leq \frac{E\|w(h)\|^{4}}{\frac{r^{4}}{81}} \leq c \frac{h^{2}}{r^{4}},
$$

where $c>0$ is a constant independent of $h$ and $r$.

Using now (1.10), (1.19), (1.20) and the fact that $x_{0}^{h}$ is a maximum point 
of $\left(v_{h}-\varphi\right)$ on $\bar{B}\left(x_{0}, r\right)$ we obtain

$$
\begin{aligned}
0 & =v_{h}\left(x_{0}^{h}\right)+\sup _{a \in A}\left\{\int _ { \Omega _ { r } } \left[-(1-\lambda h) v_{h}\left(x_{0}^{h}+h b\left(x_{0}^{h}, a\right)+\sqrt{\varepsilon} w(h)\right)\right.\right. \\
& \left.-h f\left(x_{0}^{h}, a\right)\right] d P+\int_{\Omega \backslash \Omega_{r}}\left[-(1-\lambda h) v_{h}\left(x_{0}^{h}+h b\left(x_{0}^{h}, a\right)+\sqrt{\varepsilon} w(h)\right)\right. \\
& \left.-h f\left(x_{0}^{h}, a\right)\right] d P \geq \sup _{a \in A}\left\{\int _ { \Omega _ { r } } \left[\varphi\left(x_{0}^{h}\right)-\varphi\left(x_{0}^{h}+h b\left(x_{0}^{h}, a\right)\right.\right.\right. \\
& \left.+\sqrt{\varepsilon} w(h))+\lambda h v_{h}\left(x_{0}^{h}+h b\left(x_{0}^{h}, a\right)+\sqrt{\varepsilon} w(h)\right)\right] d P \\
& +\int_{\Omega \backslash \Omega_{r}}\left[v_{h}\left(x_{0}^{h}\right)-(1-\lambda h) v_{h}\left(x_{0}^{h}+h b\left(x_{0}^{h}, a\right)\right.\right. \\
& \left.+\sqrt{\varepsilon} w(h))] d P-h f\left(x_{0}^{h}, a\right)\right\} .
\end{aligned}
$$

Taking into account (1.21) and the boundness of $\varphi$ and $v_{h}$, the relation (1.22) implies

$$
\begin{aligned}
0 & \geq \sup _{a \in A} E\left[\varphi\left(x_{0}^{h}\right)-\varphi\left(x_{0}^{h}+h b\left(x_{0}^{h}, a\right)+\sqrt{\varepsilon} w(h)\right)\right. \\
& \left.+\lambda h v_{h}\left(x_{0}^{h}+h b\left(x_{0}^{h}, a\right)+\sqrt{\varepsilon} w(h)\right)-h f\left(x_{0}^{h}, a\right)\right]-c_{1} \frac{h^{2}}{r^{4}},
\end{aligned}
$$

where $c_{1}>0$ is a constant independent of $h$.

Because $\varphi \in C^{2}\left(\mathbb{R}^{n}\right)$, using Itô formula, the relation (1.23) gives

$$
\begin{aligned}
0 & \geq \sup _{a \in A} E\left\{-\int_{0}^{h}\left[\nabla \varphi\left(x_{0}^{h}+s b\left(x_{0}^{h}, a\right)+\sqrt{\varepsilon} w(s)\right) \cdot b\left(x_{0}^{h}, a\right)\right.\right. \\
& \left.-\frac{\varepsilon}{2} \Delta \varphi\left(x_{0}^{h}+s b\left(x_{0}^{h}, a\right)+\sqrt{\varepsilon} w(s)\right)\right] d s-\int_{0}^{h} \nabla \varphi\left(x_{0}^{h}+s b\left(x_{0}^{h}, a\right)\right. \\
& +\sqrt{\varepsilon} w(s)) d w(s)+\lambda h v_{h}\left(x_{0}^{h}+h b\left(x_{0}^{h}, a\right)+\sqrt{\varepsilon} w(h)\right) \\
& \left.-h f\left(x_{0}^{h}, a\right)\right\}-c_{1} \frac{h^{2}}{r^{4}} .
\end{aligned}
$$

Dividing (1.24) by $h$ and using the fact that $\varphi \in C^{2}\left(\mathbb{R}^{n}\right), v_{h} \rightarrow u$ (locally uniformly as $h \rightarrow 0$ ), and $x_{0}^{h} \rightarrow x_{0}$ as $h \rightarrow 0$, it results

$$
\lambda u\left(x_{0}\right)+\sup _{a \in A}\left[-\nabla \varphi\left(x_{0}\right) \cdot b\left(x_{0}, a\right)-f\left(x_{0}, a\right)\right]-\frac{\varepsilon}{2} \Delta \varphi\left(t_{0}\right) \leq 0,
$$

i.e. $u(\cdot)$ is a viscosity subsolution of (1.1) (see Definition (1.1)). 
In a similar way one can prove that $u(\cdot)$ is also a viscosity supersolution of (1.1). In our hypotheses on $\lambda, b, f$ the equation (1.1) has an unique viscosity solution given by $J(\cdot)$ (see $[7])$. So $u(\cdot)=J(\cdot)$ and Theorem 1.3 is proved.

\section{The rate of convergence of the approximation scheme}

In this section we shall prove a result with respect to the rate of convergence of the approximate solutions $v_{h}(\cdot)$ to the viscosity solution $J(\cdot)$ of equation (1.1) and also we shall improve the result in Theorem 1.3. Such results for the deterministic case were given in [1], [3], [5].

Theorem 2.1. In the hypotheses $(\mathrm{H})$ we have

$$
\sup _{x \in \mathbb{R}^{n}}\left|J(x)-v_{h}(x)\right| \leq c h, 0<h \leq \frac{1}{\lambda},
$$

where $c>0$ is a constant independent of $h$.

Proof. Let $\delta, \rho \in(0,1)$ be fixed. We define the auxiliary function

$$
\varphi(x, y)=v_{h}(x)-J(y)-\frac{\delta\|x-y\|^{2}}{\rho^{2}},(x, y) \in \mathbb{R}^{n} \times \mathbb{R}^{n}, 0<h \leq \frac{1}{\lambda}
$$

Using the fact that $v_{h}$ and $J$ are bounded (see (1.19) and (1.3)) it results that for each $\gamma \in(0,1)$ there exists $\left(x_{1}, y_{1}\right) \in \mathbb{R}^{n} \times \mathbb{R}^{n}$ such that

$$
\varphi\left(x_{1}, y_{1}\right)>\sup _{(x, y) \in \mathbb{R}^{n} \times \mathbb{R}^{n}} \varphi(x, y)-\gamma .
$$

Let's consider a smooth function $\xi \in C_{0}^{\infty}\left(\mathbb{R}^{n} \times \mathbb{R}^{n}\right)$ which satisfies

$$
\xi\left(x_{1}, y_{1}\right)=1,0 \leq \xi \leq 1,\|\nabla \xi\| \leq 1 .
$$

Define

$$
\psi(x, y)=\varphi(x, y)+\gamma \xi(x, y),(x, y) \in \mathbb{R}^{n} \times \mathbb{R}^{n} .
$$

From (2.2) and (2.4) we obtain that there exists $\left(x_{0}, y_{0}\right) \in \mathbb{R}^{n} \times \mathbb{R}^{n}$ such that

$$
\psi\left(x_{0}, y_{0}\right) \geq \psi(x, y),(x, y) \in \mathbb{R}^{n} \times \mathbb{R}^{n} .
$$


So, the function $-\psi\left(x_{0}, y\right)=J(y)-\left(v_{h}\left(x_{0}\right)-\delta \frac{\left\|x_{0}-y\right\|^{2}}{\rho^{2}}+\gamma \xi\left(x_{0}, y\right)\right)$ has a global minimum point in $y_{0}$. Using the fact that $J(\cdot)$ is a viscosity supersolution of (1.1) it results (see Definition 1.1)

$$
\begin{aligned}
& \lambda J\left(y_{0}\right)+\sup _{a \in A}\left[-\frac{2 \delta}{\rho^{2}}\left(x_{0}-y_{0}\right) \cdot b\left(y_{0}, a\right)-\gamma\left(\nabla_{y} \xi\left(x_{0}, y_{0}\right)\right) \cdot b\left(y_{0}, a\right)\right. \\
& \left.-f\left(y_{0}, a\right)\right]+\varepsilon \frac{\delta}{\rho^{2}}-\frac{\varepsilon}{2} \gamma \Delta_{y} \xi\left(x_{0}, y_{0}\right) \geq 0 .
\end{aligned}
$$

The set $A$ being compact it results that there exists $a_{0} \in A$ such that (see $(2.6))$

$$
\begin{aligned}
& \lambda J\left(y_{0}\right)-\frac{2 \delta}{\rho^{2}}\left(x_{0}-y_{0}\right) \cdot b\left(y_{0}, a_{0}\right)-\gamma\left(\nabla_{y} \xi\left(x_{0}, y_{0}\right)\right) \cdot b\left(y_{0}, a_{0}\right) \\
& -f\left(y_{0}, a_{0}\right)+\varepsilon \frac{\delta}{\rho^{2}}-\frac{\varepsilon}{2} \gamma \Delta_{y} \xi\left(x_{0}, y_{0}\right) \geq 0 .
\end{aligned}
$$

The function $v_{h}(\cdot)$ verifies the relation (1.17) and so we have

$$
v_{h}\left(x_{0}\right)-(1-\lambda h) E v_{h}\left(x_{0}+h b\left(x_{0}, a_{0}\right)+\sqrt{\varepsilon} w(h)\right)-h f\left(x_{0}, a_{0}\right) \leq 0 .
$$

Using (2.5) we obtain

$$
\begin{aligned}
v_{h}\left(x_{0}\right) & -J\left(y_{0}\right)-\frac{\delta\left\|x_{0}-y_{0}\right\|^{2}}{\rho^{2}}+\gamma \xi\left(x_{0}, y_{0}\right) \\
& \geq E\left[v_{h}\left(x_{0}+h b\left(x_{0}, a_{0}\right)+\sqrt{\varepsilon} w(h)\right)\right. \\
& -\frac{\delta\left\|x_{0}+h b\left(x_{0}, a_{0}\right)+\sqrt{\varepsilon} w(h)-y_{0}\right\|^{2}}{\rho^{2}} \\
& \left.+\gamma \xi\left(x_{0}+h b\left(x_{0}, a_{0}\right)+\sqrt{\varepsilon}(h), y_{0}\right)\right]-J\left(y_{0}\right),
\end{aligned}
$$

i.e.

$$
\begin{aligned}
E v_{h}\left(x_{0}\right. & \left.+h b\left(x_{0}, a_{0}\right)+\sqrt{\varepsilon} w(h)\right) \\
& \leq v_{h}\left(x_{0}\right)+\gamma \xi\left(x_{0}, y_{0}\right)-\frac{\delta\left\|x_{0}-y_{0}\right\|^{2}}{\rho^{2}} \\
& +\frac{\delta}{\rho^{2}} E\left\|x_{0}-y_{0}+h b\left(x_{0}, a_{0}\right)+\sqrt{\varepsilon} w(h)\right\|^{2} \\
& -\gamma E \xi\left(x_{0}+h b\left(x_{0}, a_{0}\right)+\sqrt{\varepsilon} w(h), y_{0}\right) .
\end{aligned}
$$


The relations (2.8) and (2.9) imply

$$
\begin{aligned}
& \lambda h v_{h}\left(x_{0}\right)-(1-\lambda h) \gamma \xi\left(x_{0}, y_{0}\right)+\frac{(1-\lambda h) \delta}{\rho^{2}}\left\|x_{0}-y_{0}\right\|^{2} \\
& -\frac{(1-\lambda h) \delta}{\rho^{2}} E\left\|x_{0}-y_{0}+h b\left(x_{0}, a_{0}\right)+\sqrt{\varepsilon} w(h)\right\|^{2} \\
& +(1-\lambda h) \delta E \xi\left(x_{0}+h b\left(x_{0}, a_{0}\right)+\sqrt{\varepsilon} w(h), y_{0}\right)-h f\left(x_{0}, a_{0}\right) \leq 0 .
\end{aligned}
$$

Dividing (2.10) by $h$ we obtain

$$
\begin{aligned}
& \lambda v_{h}\left(x_{0}\right)-\frac{1-\lambda h}{h} \gamma \xi\left(x_{0}, y_{0}\right)+\frac{(1-\lambda h) \delta}{h \rho^{2}}\left\|x_{0}-y_{0}\right\|^{2} \\
& -\frac{(1-\lambda h) \delta}{h \rho^{2}} E\left\|x_{0}-y_{0}+h b\left(x_{0}, a_{0}\right)+\sqrt{\varepsilon} \omega(h)\right\|^{2} \\
& +\frac{(1-\lambda h) \delta}{h} E \xi\left(x_{0}+h b\left(x_{0}, a_{0}\right)+\sqrt{\varepsilon} w(h), y_{0}\right)-f\left(x_{0}, a_{0}\right) \leq 0 .
\end{aligned}
$$

Using the hypotheses $(\mathrm{H})$ and also the properties of $\xi$ and $w(h)(E w(h)=0$, $\left.E\|w(h)\|^{2}=h\right)$, the inequality (2.11) implies

$$
\begin{aligned}
\lambda v_{h}\left(x_{0}\right) & -\frac{\gamma}{h}-\frac{(1-\lambda h) \delta h M^{2}}{\rho^{2}} \\
& -\frac{(2-\lambda h) \delta}{\rho^{2}} \varepsilon-\frac{2 \delta}{\rho^{2}}\left(x_{0}-y_{0}\right) \cdot b\left(x_{0}, a_{0}\right) \\
& -\frac{2 \lambda h \delta}{\rho^{2}} M\left\|x_{0}-y_{0}\right\|-\frac{h \delta}{\rho^{2}}-f\left(x_{0}, a_{0}\right) \leq 0 .
\end{aligned}
$$

Subtracting (2.7) from (2.12) it results

$$
\begin{aligned}
\lambda\left(v_{h}\left(x_{0}\right)\right. & \left.-J\left(y_{0}\right)\right)+\gamma\left(\nabla_{y} \xi\left(x_{0}, y_{0}\right)\right) b\left(y_{0}, a_{0}\right)-\frac{\gamma}{h} \\
& -\frac{(1-\lambda h)}{\rho^{2}} \delta h M^{2}-\frac{(3-\lambda h) \delta}{\rho^{2}} \varepsilon-\frac{2 \lambda h \delta}{\rho^{2}} M\left\|x_{0}-y_{0}\right\| \\
& -\frac{h \delta}{\rho^{2}}+\frac{\varepsilon}{2} \gamma \Delta_{y} \xi\left(x_{0}, y_{0}\right) \leq 0 .
\end{aligned}
$$

Making $x=y=x_{0}$ in relation (2.5) and using the properties of $v_{h}$ and $\xi$ (see (1.19) and (2.3)) it results

$$
\delta\left\|x_{0}-y_{0}\right\| \leq\left(\gamma+\frac{L_{2}}{\lambda-L_{1}}\right) \rho^{2} .
$$


Taking again into account hypotheses $(\mathrm{H})$ and (2.3), the relations (2.13) and (2.14) imply

$$
v_{h}\left(x_{0}\right)-J\left(y_{0}\right) \leq c\left(\gamma+\frac{\gamma}{h}+\frac{h \delta}{\rho^{2}}+\frac{\delta}{\rho^{2}}+h\right),
$$

where $c>0$ is a constant independent of $h, \rho, \gamma$, and $\delta$.

Now taking $\gamma=h^{2}, \delta=h^{3}, \rho=h$, the relation (2.15) gives

$$
v_{h}\left(x_{0}\right)-J\left(y_{0}\right) \leq c h \text { for } 0<h \leq \frac{1}{\lambda} .
$$

Putting in the relation (2.5) $y=x$, it results

$$
v_{h}(x)-J(x)+\gamma \xi(x, x) \leq v_{h}\left(x_{0}\right)-J\left(y_{0}\right)-\frac{\delta\left\|x_{0}-y_{0}\right\|^{2}}{\rho^{2}}+\gamma \xi\left(x_{0}, y_{0}\right)
$$

from where, using (2.16), we obtain

$$
v_{h}(x)-J(x) \leq c h, x \in \mathbb{R}^{n}, 0<h \leq \frac{1}{\lambda},
$$

where $c>0$ is a constant independent of $h$.

To prove the opposite inequality we consider the auxiliary function

$$
\widetilde{\varphi}(x, y)=J(y)-v_{h}(x)+\frac{\delta\|x-y\|^{2}}{\rho^{2}}
$$

which is bounded from below.

Using, this time, the fact that $J(\cdot)$ is a viscosity subsolution of (1.1) and making a similar calculus as before we obtain the desired result.

Remark 2.1. Theorem 2.1 established that the approximate solution $v_{h}(\cdot)$ converges globally uniformly on $\mathbb{R}^{n}$ to the viscosity solution $J(\cdot)$ and so we have obtained an improvement of the result in Theorem 1.3 (see [6]).

\section{REFERENCES}

1. BARdi, M.; FALCONE, M. - An approximation scheme for the minimum time function, SIAM J. Control Optim., 28 (1990), 950-965. 
2. Bensoussan, A. - Stochastic Control by Functional Analysis Methods, Studies in Mathematics and its Applications, North-Holland Publishing Co., Amsterdam-New York, 1982.

3. Capuzzo Dolcetta, I. - On a discrete approximation of the Hamilton-Jacobi equation of dynamic programming, Appl. Math. Optim., 10 (1983), 367-377.

4. Capuzzo-Dolcetta, I.; Falcone, M. - Discrete dynamic programming and viscosity solutions of the Bellman equation, Analyse non linéaire, Ann. Inst. H. Poincaré Anal. Non Linéaire, 6 (1989), 161-183.

5. Capuzzo-Dolcetta, I.; Ishis, H. - Approximate solutions of the Bellman equation of deterministic control theory, Appl. Math. Optim., 11 (1984), 161-181.

6. HaVÂRNEANU, T. - An approximation scheme of the dynamic programming equation for a stochastic control problem with infinite horizon, Differential equations and control theory (Iasi, 1990), 103-111, Pitman Res. Notes Math. Ser., 250, Longman Sci. Tech., Harlow, 1991.

7. Lions, P.-L. - Optimal control of diffusion processes and Hamilton-Jacobi-Bellman equations. II, Viscosity solutions and uniqueness, Comm. Partial Differential Equations, 8 (1983), 1229-1276.

Received: 15.X.2009

Department of Mathematics,

University "Al.I. Cuza", 11, Bd. Carol I, 700506, Iaşi,

ROMANIA

and

Institute of Mathematics "Octav Mayer", Romanian Academy, Iaşi Branch,

ROMANIA

havi@uaic.ro 